\title{
Insight on Bone Morphogenetic Protein 7 in Ankylosing Spondylitis and its association with disease activity and radiographic damage
}

\author{
Adel Mahmoud ${ }^{1}$, Dalia Fayez ${ }^{1}$, Mervat Mammdouh Abou Gabal ${ }^{1}$, Sherin Mohamed Hosny Hamza ${ }^{1}$, Takwa Badr ${ }^{2}$ \\ ${ }^{1}$ Internal Medicine \& Rheumatology Departments, Faculty of Medicine, Ain Shams University, Cairo, Egypt \\ ${ }^{2}$ Physical Medicine, Rheumatology and Rehabilitation Departments, Faculty of Medicine, Ain Shams University, \\ Cairo, Egypt
}

\section{Type of article: Original}

\begin{abstract}
Introduction: Fusion of joints as well as intervertebral spaces by the formation of bony spurs appearing as syndesmophytes and osteophytes are the hallmark of spondyloarthropathies which accounts for disability. The aim of this study was to assess the serum level of bone morphogenetic protein (BMP)-7 in ankylosing spondylitis and its relationship with disease activity and the radiographic damage.

Methods: This longitudinal case control study was conducted in Ain Shams University Hospitals (Egypt). A total of 55 subjects were included in two case groups and one control group. Group I included 20 patients with Ankylosing Spondylitis (AS) assessed at baseline (defined as Ia and after 18 months defined as Ib). Group II included 20 patients with Rheumatoid Arthritis (RA) and Group III included 15 healthy subjects as controls. Patients with other forms of seronegative spondyloarthropathies, bone forming diseases were excluded from the study. The Bath Ankylosing Spondylitis Disease Activity Index (BASDAI) and the Bath Ankylosing Spondylitis Metrology Index (BASMI) were used to assess disease activity in AS patients. RA disease activity was assessed using the disease activity score 28 (DAS28). Radiographic changes were assessed using the Bath AS Radiographic Index (BASRI) in AS and Larsen scores in RA. Laboratory investigations included: Complete blood picture (CBC), Erythrocyte sedimentation rate (ESR), quantitative CRP, serum calcium, phosphorus and alkaline phosphatase. Determination of serum bone morphogenetic protein-7 level (BMP-7) was done using enzyme linked immunosorbent assay (ELISA). Sample collections, clinical and radiological assessments were performed at baseline for all groups and after a mean follow-up of 18 months for Group I. Data were analyzed by SPSS 17, using t-test, Kruskal-Wallis, Mann-Whitney, Fischer exact test, Chi square, and Pearson ProductMoment Correlation Coefficient.

Results: There were statistically significant differences between the 3 groups as regard baseline BMP-7 levels; the mean BMP-7 level of AS patients was significantly higher than that of RA patients and controls and significantly higher in the RA group than that of controls. BMP-7 levels were not associated with any of the clinical or drug related variables either in AS or RA. In AS BMP-7 levels showed significant increase after follow up and significant positive correlation with serum alkaline phosphatase (both at baseline and after follow up) and BASDAI score (after follow up) respectively. Despite the parallel increase of BMP7 and BASRI score during the follow-up period no statistically significant correlation was detected. There were no significant correlations between BMP7 level and patient's age or any disease related characteristics in the RA group.

Conclusion: A significant progressive increase in serum BMP-7 was noted in AS patients that correlated with serum markers of bone formation. Such a biomarker measurement may not only act as a surrogate marker for the disease but has the potential to contribute to the pathogenesis of AS that may provide a complementary or alternative therapeutic approach.
\end{abstract}

Keywords: Bone morphogenic protein-7, Ankylosing spondylitis, Radiographic changes

\section{Corresponding author:}

Assistant Professor Dr. Sherin Mohamed Hosny Hamza, Ain Shams University, Cairo, Egypt.

Tel.: +201223304460, E-mail: drsherinehosny@hotmail.com

Received: March 17, 2016, Accepted: May 27, 2016, Published: July 2016

iThenticate screening: May 27, 2016, English editing: June 10, 2016, Quality control: July 04, 2016

(C) 2016 The Authors. This is an open access article under the terms of the Creative Commons Attribution-NonCommercialNoDerivs License, which permits use and distribution in any medium, provided the original work is properly cited, the use is non-commercial and no modifications or adaptations are made. 


\section{Introduction}

Ankylosing spondylitis is considered as the most common form of spondyloarthritis and is due to chronic inflammation affecting the spinal joints with variable involvement of large joints in the periphery (1). Ankylosing enthesitis, is a typical characteristic of Spondyloarthropathies (SpA). The reaction causes heterotopic cartilage and the formation of new bone, which eventually causes the fusion of joints. This process is seen as the most classic symptom of $\mathrm{SpA}$. Another symptom of $\mathrm{SpA}$ is the inflammation of the synovial membrane (synovitis). Although, in appearance, $\mathrm{SpA}$ seems less severe than the more common, Rheumatoid Arthritis, (RA) SpA can lead to great suffering and disability for those afflicted with the disease. Defining the pathological process of SpA has been the subject of new medical approaches in regenerative medicine, and could in the near future, be beneficial to the variable sufferers of remodeling arthritis, which is the characteristic symptom of SpA, destructive arthritis, which is typical for RA, and also steady-state arthritis, as identified in osteoarthritis (OA) Also fitting into steady-state arthritis form, could be the non-erosive rheumatoid factor negative patients with mild RA, however, rapidly progressive OA and some forms of psoriatic arthritis may fit in the destructive arthritis group (2). Even if the control of the inflammatory process is achieved, there is still a possibility the process of joint erosion in RA or SpA, however, and this could eventually lead to secondary OA, despite the restoration of the joint homeostasis (3). The bone morphogenetic proteins which are members of the transforming growth factor- $\beta$ (TGF- $\beta$ ) superfamily, also play a crucial role in osteoblastic differentiation and function. They are important for the skeletal development and the joint morphogenesis, they appear to have a role in cartilage and bone homeostasis. BMP are identified by their unique ability to induce ectopic cartilage development and bone formation in vivo. BMP are also important in the joint remodeling of arthritic diseases, particularly in the enthesophyte formation in SpA. The immunohistochemical staining revealed active BMP signaling present in target cells of human SpA enthesitis. Moreover, the recombinant human BMP were effective in the induction of bone healing and the enhancement of spinal fusion in human trials. Overproduction of the BMP-2 and the BMP-7 in patients with AS has been reported. While the mechanism of the new bone formation is not fully defined in AS and the related SPA; the BMP seems to play a significant role in spinal ankylosis and may be of a therapeutic target (4). The aim of this study was to assess the serum level of bone morphogenetic protein (BMP) 7 in ankylosing spondylitis, and its relationship with disease activity and the radiographic damage.

\section{Material and Methods}

\subsection{Subjects}

This longitudinal case control study was carried out on 55 subjects divided into 3 groups: Group I: Included 20 patients with Ankylosing Spondylitis (AS), diagnosed according to the modified New York criteria (5). Group II included 20 age matched patients with RA, diagnosed according to the 1987 revised criteria of the American College of Rheumatology (6), they were also fulfilling the new EULAR / ACR criteria (7).Group III: Included 15 age and sex matched healthy subjects. Patients with other forms of seronegative spondyloarthropathies, bone forming diseases, primary degenerative axial and peripheral arthropathies were excluded from the study. Patients were recruited from the Rheumatology outpatient clinic or from the inpatient Unit of Rheumatology at Ain Shams University Hospitals in the period from January 2010 to December 2012. Informed consent was obtained from participants.

\subsection{Clinical evaluation}

All patients were subjected to detailed medical history taking and a thorough physical examination including a detailed musculoskeletal examination. Assessment of functional disease activity in group I (AS) was performed at baseline (Ia) and after a follow-up duration of 18 months (Ib) using Bath Ankylosing Spondylitis Disease Activity Index (BASDAI) (8), the resulting 0 to 50 score was divided by 5 to give a final 0-10 BASDAI score. Also the Bath Ankylosing Spondylitis Metrology Index was performed (BASMI) (9). It includes 5 simple clinical measurements then calculating the scores for each of the BASMI measurements. Assessment of disease activity in group II (RA) was performed using the disease activity score (DAS) 28 (10).

\subsection{Laboratory and radiological assessment}

Laboratory investigations (for all studied population) included the following: Complete blood picture (CBC) by using coulter GS+ cell counter. ESR $1^{\text {st }} \mathrm{hr}$ : using Westergren method, quantitative CRP, serum creatinine, blood urea nitrogen and complete urine analysis were done by calorimetric method, serum albumin, calcium, phosphorus and alkaline phosphatase were also measured and the results were recorded. Assays of serum BMP7 was performed using enzyme linked immunosorbent assay (ELISA) commercial method using a kit for (Human BMP-7, R\&D Systems Inc., Minneapolis, MN, USA), according to the manufacturer's instructions and carried out tests in 
duplicate. The minimum detectable dose of BMP-7 ranged from 0.79 to $7.83 \mathrm{pg} / \mathrm{ml}$. Serum samples were collected and kept frozen at $-80^{\circ} \mathrm{C}$ until use. $100 \mu \mathrm{l}$ standard or sample was added to each well, and incubated for 2.5 hours at room temperature. $100 \mu \mathrm{l}$ of prepared biotin antibody was added and incubated for 1 hour at room temperature. 100 $\mu \mathrm{l}$ prepared Streptavidin solution was added and incubated 45 minutes. $100 \mu$ TMB One-Step Substrate Reagent was added, and incubated 30 minutes. $50 \mu \mathrm{l}$ Stop Solution was added to each well. Read at $450 \mathrm{~nm}$ immediately. The mean absorbance for each set of duplicate standards, controls and samples was calculated, and subtracted the average zero standard optical density. A standard curve was constructed using a log-log graph. All AS patients underwent Cervical and lumbar plain X-ray for assessment of radiographic changes using the Bath AS Radiographic Index (BASRI), range=2-12 (11). For RA patients plain X-rays of hand and feet were done for assessment of radiographic changes using Larsen scores, 1995, the scores ranged from 0 to 160 (12).

\subsection{Statistical analysis}

The statistical program used was SPSS 17 (SPSS Inc. Chicago, Illinois, USA). Quantitative data were analyzed using mean and standard deviation, while frequency and percentage were used with qualitative data. T-tests KruskalWallis and Mann-Whitney tests were used to compare means of different groups, while Fischer exact test and chi square were used to compare frequencies. Pearson correlation was used to find relationships between variables. Results were considered significant at $\mathrm{p}$ value $<0.05$.

\subsection{Ethics of research}

The study was approved by Ain Shams Medical Ethics Committee.

\section{Results}

This longitudinal case control study included 20 patients with AS (group I), all were males, their ages ranged from 18 to 53 years with mean \pm SD of $35.000 \pm 10.795$ years. However, our selected 20 RA patients (group II) included only 9 males (45\%) and 11 females (55\%). Their ages ranged from 21 to 61 years with mean \pm SD of $40.450 \pm$ 12.927 years. As regards to the control (group III), they were 15 healthy individual and were all males and their ages ranged from 21 to 50 years with mean \pm SD of $35.66 \pm 9.861$ years. There was statistical significant difference between the three groups as regard to sex (with predominant male gender among AS and control groups). Also there was significant difference regarding the age of disease onset between AS and RA groups, being higher in RA, $\mathrm{p}<0.05$. However, there was no significant difference between the three groups or between AS and RA groups regarding age and disease duration respectively $(\mathrm{p}>0.05)$. The commonest clinical manifestations among the AS patients were peripheral arthritis in 14 patients (70\%), enthesitis in 5 patients $(25 \%)$, and uveitis in 2 patients (10\%). Comparison between AS and RA groups as regards drug intake by chi-square test showed that there was a statistically significant difference between the 2 groups as regards the use of Sulfasalazine, MTX, Leflunamide, hydroquine, Steroids and anti-TNF $(\mathrm{p}<0.05)$, the intake of MTX, lefunamide, hydroquine, and steroids were more in RA group II while, sulfasalazine and anti TNF intake were more in AS group I. However, there was no statistically significant difference between the 2 groups as regards the use of NSAIDs ( $p>0.05)$ (Table 1). Comparison between $\mathrm{AS}$ and RA groups as regards $\mathrm{CBC}$, renal function, bone metabolic markers and acute phase reactants (levels at baseline) revealed statistically significant difference between the 2 groups in hemoglobin level being lower in RA group, $p<0.05$. As regards to other laboratory findings, there wasn't a statistically significant difference ( $p>0.05$ ) (data not shown).Comparisons between AS, RA and control groups as regards the mean BMP7 levels at base line showed that there was a statistically highly significant difference between the 3 groups as regards baseline BMP-7 levels $(\mathrm{p}<0.05)$. By Mann-Whitney test the mean BMP-7 level of AS patients was significantly higher than those of RA patients and controls $(p=0.000)$, and also the mean BMP-7 level of the RA group was significantly higher than that of controls $(\mathrm{p}=0.002)$. On comparing the mean serum levels of BMP-7 in AS patients group Ia (AS patients at base line) as regards to the presence or absence of Enthesitis, Uveitis or Peripheral arthritis by unpaired $\mathrm{T}$ test results, showed that the levels were higher in patients with Enthesitis, Uveitis and Peripheral arthritis than those without, but results didn't reach a statistical significance, $(p>0.05)$ which may be due to small patients sample (data not shown). The use of TNF blockers or SSZ didn't have any statistically significant association with the serum levels of BMP-7 ( $>0.05)$ although the levels were less in patients who received these medications which may be due to the relatively short follow up period in our study (data not shown). Comparison between group1-a (AS patients at base line) and group 1-b (after a follow up period of 18 months) as regards BMP7, acute phase reactants, parameters of bone metabolism \{serum calcium (Ca), phosphorous (Po4), alkaline phosphatase (ALK.ph)\} and indices of disease activity and damage (BASDAI, BASMI, and BASRI) by unpaired test showed that there was a significant increase in the BMP7 level after the follow up period in AS patients. Moreover, there was significant increase in BASRI, and decrease in ESR and BASDAI scores respectively, after the follow up period with $\mathrm{p}<0.05$. 
However, there were no significant changes regarding other parameters $(\mathrm{p}>0.05)$. There was a statistically significant positive correlation between mean serum BMP7levels and serum alkaline phosphatase in AS group at baseline and after follow up, $\mathrm{p}<0.05$. Also there was a significant positive correlation between BMP7 and the BASDAI score only after the follow-up period, $\mathrm{p}<0.05$. There was no significant correlation between BMP7 and other parameters $\mathrm{p}>0.05$ (Tables 2, 3).

Table 1. Comparison between AS, RA, and controls groups as regards to age, age of onset, sex, disease duration, and drug intake.

\begin{tabular}{|c|c|c|c|c|c|}
\hline \multicolumn{2}{|l|}{ Variables } & AS patients & RA patients & Controls & p-value \\
\hline \multicolumn{2}{|c|}{ Age (year); Mean \pm SD } & $35 \pm 10.7$ & $40.4 \pm 12.9$ & $35.6 \pm 9.8$ & 0.27 \\
\hline \multicolumn{2}{|c|}{ Age of disease onset (year); Mean \pm SD } & $25.6 \pm 9.4$ & $33.3 \pm 12.4$ & & 0.033 \\
\hline \multicolumn{2}{|c|}{ Disease duration (year); Mean \pm SD } & $9.4 \pm 6.9$ & $7.1 \pm 5.26$ & & 0.246 \\
\hline \multirow[t]{2}{*}{ Sex; n (\%) } & Male & $20(100)$ & $9(45)$ & $15(100)$ & \multirow[t]{2}{*}{$<0.001$} \\
\hline & Female & $0(0)$ & $11(55)$ & $0(0)$ & \\
\hline \multirow[t]{7}{*}{ Drug intake; n (\%) } & Sulfasalazine & $13(65)$ & $2(10)$ & & 0.000 \\
\hline & MTX & $2(10)$ & $10(50)$ & & 0.006 \\
\hline & Leflunamide & $0(0)$ & $8(40)$ & & 0.002 \\
\hline & Hydroquine & $0(0)$ & $12(60)$ & & 0.000 \\
\hline & NSAIDs & $19(95)$ & $20(100)$ & & 0.31 \\
\hline & Steroids & $7(35)$ & $18(90)$ & & 0.000 \\
\hline & Anti-TNF & $8(40)$ & $0(0)$ & & 0.005 \\
\hline
\end{tabular}

MTX: methotrexate, NSAIDs: non-steroidal anti- inflammatory drugs, Anti-TNF: anti-tumor necrosis factor

Table 2. Comparison between group1-a and group 1-b as regards to BMP7, acute phase reactants, parameters of bone metabolism and indices of disease and damage.

\begin{tabular}{|l|l|l|l|}
\hline Variables & $\begin{array}{l}\text { Group 1-a (AS patients at base line); } \\
\text { Mean } \pm \text { SD }\end{array}$ & $\begin{array}{l}\text { Group 1-b (AS patients after follow up); } \\
\text { Mean } \pm \text { SD }\end{array}$ & $\begin{array}{l}\text { p- } \\
\text { value }\end{array}$ \\
\hline $\begin{array}{l}\text { Serum BMP7 } \\
(\mathrm{pg} / \mathrm{ml})\end{array}$ & $418.75 \pm 272.5$ & $764.2 \pm 343.6$ & 0.000 \\
\hline $\mathrm{ESR}(\mathrm{mm} / \mathrm{hr})$ & $46.5 \pm 25.741$ & $35.95 \pm 14.188$ & 0.022 \\
\hline $\mathrm{CRP}(\mathrm{mg} / \mathrm{dl})$ & $17.15 \pm 21.06$ & $13.250 \pm 10.74$ & 0.263 \\
\hline Serum $\mathrm{Ca}(\mathrm{mg} / \mathrm{dl})$ & $9.250 \pm 0.635$ & $9.315 \pm 0.709$ & 0.503 \\
\hline Serum Po $(\mathrm{mg} / \mathrm{dl})$ & $3.595 \pm 0.619$ & $3.505 \pm 0.700$ & 0.518 \\
\hline Serum ALP $(\mathrm{U} / \mathrm{L})$ & $276.250 \pm 140.8$ & $267.050 \pm 104.66$ & 0.42 \\
\hline BASDAI & $3.330 \pm 1.459$ & $2.780 \pm 1.352$ & 0.018 \\
\hline BASMI & $4.600 \pm 1.875$ & $4.850 \pm 1.814$ & 0.056 \\
\hline BASRI & $5.050 \pm 2.012$ & $6.050 \pm 1.877$ & 0.002 \\
\hline
\end{tabular}

Table 3. Correlation between BMP7 and age, disease related characteristics and parameters of bone metabolism in AS group at baseline and after follow up period.

\begin{tabular}{|c|c|c|c|c|}
\hline \multirow[t]{3}{*}{ Variables } & \multicolumn{4}{|c|}{ BMP-7 level in AS } \\
\hline & \multicolumn{2}{|c|}{ Groupla (AS patients at base line) } & \multicolumn{2}{|c|}{ Group 1b (AS patients after follow up) } \\
\hline & $\mathrm{r}$ & p-value & $\mathrm{r}$ & p-value \\
\hline Age (year) & 0.240 & 0.308 & - & - \\
\hline Age of disease onset (year) & 0.050 & 0.833 & - & - \\
\hline Disease duration (year) & 0.303 & 0.194 & - & - \\
\hline ESR $(\mathrm{mm} / \mathrm{hr})$ & 0.317 & 0.174 & 0.117 & 0.624 \\
\hline $\mathrm{CRP}(\mathrm{mg} / \mathrm{dl})$ & 0.164 & 0.490 & 0.301 & 0.197 \\
\hline Serum Ca $(\mathrm{mg} / \mathrm{dl})$ & 0.146 & 0.538 & -0.322 & 0.166 \\
\hline Serum $\mathrm{Po}_{4}(\mathrm{mg} / \mathrm{dl})$ & 0.243 & 0.301 & -0.143 & 0.547 \\
\hline Serum ALP (U/L) & 0.632 & 0.003 & 0.607 & 0.005 \\
\hline BASDAI & 0.392 & 0.088 & 0.544 & 0.01 \\
\hline BASMI & 0.259 & 0.270 & 0.263 & 0.263 \\
\hline BASRI & 0.120 & 0.614 & -0.008 & 0.975 \\
\hline
\end{tabular}


The ratio of change of BMP-7 levels did not show any significant correlation with that of BASDAI, BASMI or BASRI scores, $\mathrm{P}>0.05$ (data not shown). Comparison between RA patients with and without drug intake (steroids and other DMARDS) as regard BMP-7 levels, no statistical significant difference was detected, $p>0.05$ (data not shown). Moreover, there was no significant correlation between BMP7 level and patients' age or any of the disease related characteristics in RA group, $\mathrm{p}>0.05$ (Table 4).

Table 4. Correlation between BMP7 with patients, and disease related characteristics in RA group.

\begin{tabular}{|l|l|l|}
\hline \multirow{2}{*}{ Group II (RA patients) } & \multicolumn{3}{|l|}{ BMP-7 } \\
\cline { 2 - 3 } & $\mathrm{r}$ & $\mathrm{p}$-value \\
\hline Age (year) & -0.005 & 0.982 \\
\hline Disease duration (year) & -0.265 & 0.258 \\
\hline Age of disease onset (year) & 0.107 & 0.655 \\
\hline ESR (mm/hr) & 0.139 & 0.559 \\
\hline CRP(mg/dl) & 0.153 & 0.519 \\
\hline DAS28 & 0.033 & 0.891 \\
\hline Larsen score & -0.257 & 0.274 \\
\hline
\end{tabular}

\section{Discussion}

Ankylosing spondylitis (AS) is a form of spondyloarthritis ( $\mathrm{SpA}$ ) which has a strong genetic association with human leukocyte antigen B27 (HLA-B27). The structural 'damage' of the spine which includes; vertebral squaring, the formation of syndesmophytes, bony erosions and joint ankylosis, are all considered to be a hallmark of AS (13). The relationship between tissue inflammation and the subsequent vertebral ankylosis still remains unclear (4). It is likely that both the mechanical and the inflammatory components of the disease, locally trigger the increase in the new bone formation (14).Although the mechanism of that new bone formation is not fully defined in AS and related SpA; BMP are likely to play an important role in spinal ankylosis and could be possible therapeutic targets. It was also shown that BMP-7 stimulates the synthesis of both proteoglycans and collagen (4). After all that has been written and said about the disease, one can confidently surmise that there are two stages to the process of ankylosing spondylitis. In the first stage, erosion of the bone's structure, which has been triggered by inflammation, causes defects and are filled with fibrous tissue. In the second stage, ossification occurs. Once inflammation has dissipated, ossification becomes much more effective; however, this is not always straight forward as inflammation can recur and disrupt the ossification process many times. Regarding rheumatoid arthritis, inflammation is consistent at any given joint, so, formation of the bone was initially thought to occur once inflammation had receded. New bone formation, however, is independent from inflammation at these later stages of the disease. In our study, it was interesting to note the significant elevation of baseline BMP-7 in both AS and RA patients compared with healthy controls. Also the mean BMP-7 level of the AS patient group was significantly higher than those levels of the RA patients' group $(\mathrm{p}=0.000)$. Our results agree with the results of Park et al. (16) who reported that BMP-7 elevation was more marked in the AS group than in the RA group, also our results go with the results of kong et al. (17) who found that the serum level of BMP-7 in AS patients with and without syndesmophyte was significantly higher than in the healthy control group. We reported a significant increase in levels of BMP-7 in our AS patients follow up group (1-b after 18 months) compared to initial BMP level measures. We also reported a significant increase in BMP-7 levels in our AS patients follow up group after 18 months (group 1b), compared to initial serum level measurements. It also agrees with the results of Park et al. (18) who reported significant increase in BMP-7 level in AS patients compared to RA patients and controls and elevated serum BMP-7 levels in AS, after the follow-up period as compared to the initial measurements. However, according to their study, and in contrast to our results there was no significant difference between the RA group and controls, regarding the mean BMP-7 level. Moreover, Chen et al., (4) reported higher serum levels of BMP-2, BMP-4, and BMP-7 in AS patients compared to healthy controls; but the elevation of BMP-2 and BMP-7 did not reach statistical significance. Wendling et al., (19) found elevated serum levels of BMP-7 in patients with AS. However, there was no significant difference noted between AS patients and controls in their study in contrast to our work. All of our AS patients were males and the same was observed in the control group. However our RA patients were only 9 males (45\%) and 11 females (55\%) which significantly differed from AS and control groups. Despite being homogenous as regard their mean ages with no significant difference in between, there was significant difference between AS and RA patients as regard the age of disease onset. Our results are in agreement with that of Son and Cha (20) and Jacobs and Fehlings, (21) who found that AS is more prevalent in males than females and often starts in young adults, with the age of onset being 30 years in $80 \%$ of patients. Also, Klareskog et al. (22) reported that RA is a disease of adult patients mainly in the 
fourth to sixth decade and has a higher prevalence in women than in men. In the present study, the level of serum BMP7at base line showed no significant correlation with current age, age of disease onset, or disease duration in either AS or RA patients. This was in agreement with Park et al. (18). However, Chen et al. (4) reported significantly elevated serum BMP7 levels in AS patients with spinal fusion (who were of older age and had longer disease duration) compared to patients without spinal fusion. The difference may be due to the enrollment of patient populations with different disease stages, or different spinal severity.

In the present study, at baseline we couldn't find any association or correlation between serum BMP7 level and clinical presenting features in AS (Enthesitis, Uveitis or Peripheral arthritis), different drug intake in either AS or RA patients, and the use of TNF blockers or SSZ did not have any significant association with BMP7 level. We couldn't assess the association with NSAIDs as 19/20 of AS patients were NSAIDs users. Wendling et al. (23) found that the level of BMP-7 didn't correlate with any of the disease-related laboratory variables (ESR, CRP). Also, Park et al. (18) found no correlation between BMP7 with biochemical values and clinical variables at baseline, or after the follow-up period. In the Chen et al. study (4), both BMP and the inflammatory measures (ESR, CRP) were higher in patients with spinal fusion than in those without spinal fusion, but they did not have any correlation. This suggests that part of the regulation of BMP is inflammation independent. Spine radiograph scores had an association with serum BMP and CRP. Inflammation and bone formation may be linked in some way in patients with AS; however, they can be an independent phenomena. Park et al. (18) found that, the uses of NSAIDs or SSZ did not have any significant association with the changes in either the biochemical values or clinical parameters in patients with AS, this was in agreement with our study. By determining serum biomarkers of bone destruction and new bone formation in ankylosing spondylitis patients, and regarding the interaction between inflammation and new bone formation with and without therapeutic interventions, more information has been sought through numerous investigations (24). Bone metabolism markers suggest a positive regulatory effect on the physiological processes of alkaline phosphatase activity in AS patients treated with anti-TNF. Whether this increase is caused by enhanced trabecular bone formation to restore inflammation-induced general bone loss or by the specific development of syndesmophytes, is not fully understood (25). As regard to disease activity and radiographic changes in AS, our study revealed a significant decrease in the ESR and BASDAI score and a significant increase in the BASRI score, $\mathrm{p}<0.05$ after follow up, however there was no significant changes regarding the BASMI score. Our results also reported a significant positive correlation between the BASRI score with disease duration and serum alkaline phosphatase level $(\mathrm{p}<0.05)$. It was also noted that the radiographic score is increased, parallel to the increase of BMP7 during the follow-up period, but with no significant correlation. However, significant positive correlation was found between BMP7 and serum alkaline phosphatase (a marker of bone formation) both at base and after follow up. Also our study revealed significant positive correlation between BMP-7 levels and the BASDAI score after follow up $(\mathrm{p}<0.05)$. It was noted that the ratio of the changes of BMP-7 levels didn't correlate with that of BASDAI or BASRI. Our results are somewhat different from the Park et al. (16) study which documented significant correlation between BMP-7 levels with the BASRI (which takes syndesmophytes into account). BMP-7 variations over a mean follow-up of more than 50 months correlated with BASRI variations $(\mathrm{P}<0.05)$. These data suggest closer involvement of BMP-7 in entheseal ossification than in systemic bone turnover in patients with AS. However, Wendling et al. (23) found no correlation between BMP-7 with any of the disease related clinical variables (BASDAI, The Bath Ankylosing Spondylitis Functional Index (BASFI) and the Bath Ankylosing Spondylitis Patient Global Score (BAS-G). Park et al. (18) reported that: BMP-7 levels correlated well with BASRI-spine $(\mathrm{p}=0.01)$. BMP-7 levels significantly increased compared to initial measurements $(\mathrm{p}<0.05)$ and the changes in BMP7 levels correlated well with those of BASRI-spine in both cross-sectional and longitudinal protocols $(\mathrm{p}<0.05)$. Also the ratios of the changes of BMP-7 levels did not correlate with either BASDAI or BASRI. Absence of a correlation between BMP-7 and disease activity was noted in this study. Chen et al. (4) did not find any correlation between BMP-7, and BASDAI. BMP-7 had a correlation with the modified Stoke Ankylosing Spondylitis Spine Score (mSASSS), but not with the BASMI score. In their study, serum BMP-7 levels showed significant correlations with hip joint involvement. Elevation of BMP in serum of AS patients with spinal fusion and the positive correlation between BMP and spine BASRI and mSASSS scores all these support the notion of BMP playing an important role in the pathogenesis of spinal ankylosis in these patients. The serum BMP levels may reflect radiographic progression of the spine and hip joints. This suggests that there is significant elevation of BMP-7 in AS, but not in RA, and its strong relationship to radiographic damage of AS. The hypothesis, BMP-7 may participate in the pathogenic mechanisms which are responsible for new bone formation of the spine in AS, is supported by these data (26). The lack of significant correlation between BMP-7 levels and the BASRI score during the follow-up period in our study may be due to the short follow up period (18 months), which is not sufficient for appearance of significant radiographic changes in the plain x-rays of sacroiliac and lumbosacral joints, and also may be the effectiveness of 
biologic treatment in delaying disease progression and subsequent deterioration, as 8 out of our 20 AS patients $(40 \%)$ were on biologic treatment. Maksymowych et al. (27) reported that development of new syndesmophytes on radiography, needs nearly 2 years of follow-up. Also, radiography lacks sensitivity, so that only 5 to $10 \%$ of patients with longstanding AS, had at least one erosion and only a minority of patients had syndesmophytes extending over multiple vertebrae (28). Three main conclusions were reported by Maksymowych et al. (27): First, the presence of an active inflammatory lesion at the vertebral corner on the MRI STIR sequence was associated with the subsequent development of new syndesmophytes at the corresponding vertebral corner, which are visible on plain radiography. Second, new syndesmophytes will develop from a prior active inflammatory lesion even when there is agreement between readers that the lesion has completely resolved on follow up MRI following the initiation of anti-TNF therapy. Third, a new syndesmophyte may also develop where both the baseline plain radiograph and the baseline MRI show a completely normal vertebra. However, even though MRI shows what appears to be complete resolution of inflammation, it is possible that inflammation is still present at a histopathologic level, and this may be associated with the development of syndesmophytes. Consequently the development of new syndesmophytes in vertebrae that appear normal on MRI and plain radiography at baseline suggests either the presence of underlying inflammation that is undetectable by MRI or a role for a non-inflammatory pathway. Firstly, the growth of new syndesmophytes at the corresponding vertebral corner can be associated with the presence of an active inflammatory lesion at the vertebral corner on the MRI STIR sequence. Secondly, even when there is agreement between readers that the lesion has made full improvement on follow up MRI after anti-TNF therapy, new syndesmophytes can develop from a previous active inflammatory lesion. Thirdly, where both the baseline MRI and the baseline plain radiograph may show a completely normal vertebra, a new syndesmophyte may also develop. It is conceivable that at a histopathologic level, inflammation is still present, even though MRI shows the inflammation to be completely treated and this could be related to syndesmophyte growth. Subsequently, regarding syndesmophyte growth in vertebrae, which on MRI, and plain radiography at baseline seem normal, it is suggested that either an undetectable underlying inflammation is present or the possibility of a non-inflammatory pathway. These conclusions go with our findings of the significant positive correlation between BMP-7 levels and the BASDAI score after 18 months where, the significant decrease in ESR and the BASDAI score after 18 months, yet the residual inflammatory activity allowed new bone formation and more radiographic damage. By that time the residual low grade inflammation correlated positively with BMP-7 levels. The same results were reported by Baraliakos et al. (29).

\section{Conclusions}

In conclusion significant progressive increase in serum BMP-7 was noted in AS patients that correlated with markers of bone formation. Such biomarker measurement may not only act as a surrogate marker for the disease, but has the potential to contribute to the pathogenesis of AS which may provide a complementary or alternative therapeutic approach.

\section{Acknowledgments:}

We would like to thank our senior professors at Ain Shams University for their guidance and continuous support which helped us to accomplish this research.

\section{Conflict of Interest:}

There is no conflict of interest to be declared.

\section{Authors' contributions:}

All authors contributed to this project and article equally. All authors read and approved the final manuscript.

\section{References:}

1) Haroon N. Ankylosis in ankylosing spondylitis: current concepts. Clin Rheumatol. 2015; 34(6): 1003-7. doi: 10.1007/s10067-015-2956-4. PMID: 25935456.

2) Bay-Jensen AC, Wichuk S, Byrjalsen I, Leeming DJ, Morency N, Christiansen C, et al. Circulating protein fragments of cartilage and connective tissue degradation are diagnostic and prognostic markers of rheumatoid arthritis and ankylosing spondylitis. PLoS One. 2013; 8(1): 54504. doi: 10.1371/journal.pone.0054504. PMID: 23365672, PMCID: PMC3554760.

3) Beyer C, Schett G. Pharmacotherapy: concepts of pathogenesis and emerging treatments. Novel targets in bone and cartilage. Best Pract Res Clin Rheumatol. 2010; 24(4): 489-96. doi: 10.1016/j.berh.2010.03.001. PMID: 20732647. 
4) Chen HA, Chen CH, Lin YJ, Chen PC, Chen WS, Lu CL, et al. Association of bone morphogenetic proteins with spinal fusion in ankylosing spondylitis. J Rheumatol. 2010; 37(10): 2126-32. doi: 10.3899/jrheum.100200. PMID: 20682677.

5) Van der Linden S, Valkenburg HA, Cats A. Evaluation of diagnostic criteria for ankylosing spondylitis. A proposal for modification of the New York criteria. Arthritis Rheum. 1984; 27: 361-8. doi: 10.1002/art.1780270401. PMID: 6231933.

6) Arnett FC, Edworthy SM, Bloch DA, McShane DJ, Fries JF, Cooper NS, et al. The American Rheumatism Association 1987 revised criteria for the classification of rheumatoid arthritis. Arthritis Rheum. 1988; 31(3): 315-24. doi: 10.1002/art.1780310302. PMID: 3358796.

7) Aletaha D, Neogi T, Silman AJ, Funovits J, Felson DT, Bingham CO 3rd, et al. Rheumatoid arthritis classification criteria: an American College of Rheumatology/European League Against Rheumatism collaborative initiative. Ann Rheum Dis. 2010; 69(9): 1580-8. doi: 10.1136/ard.2010.138461. PMID: 20699241.

8) Garrett S, Jenkinson T, Kennedy LG, Whitelock H, Gaisford P, Calin A. A new approach to defining disease status in ankylosing spondylitis: the Bath Ankylosing Spondylitis Disease Activity Index. J Rheumatol. 1994; 21(12): 2286-91. PMID: 7699630.

9) Jenkinson TR, Mallorie PA, Whitelock HC, Kennedy LG, Garrett SL, Calin A. Defining spinal mobility in ankylosing spondylitis (AS). The Bath AS Metrology Index. J Rheumatol. 1994; 21(9): 1694-8. PMID: 7799351.

10) Fransen J, Van Riel PL. The Disease Activity Score and the EULAR response criteria. Clin Exp Rheumatol. 2005; 23(5Suppl 39): 93-9. PMID: 16273792.

11) Mackay K, Mack C, Bkophy S, Calin A. the Bath Ankylosing Spondylitis Radiographic Index (BASRI). Arthritis Rheum. 1998; 41(12): 2263-70. doi: 10.1002/1529-0131(199812). PMID: 9870884.

12) Boini S, Guillemin F. Radiographic scoring methods as outcome measures in rheumatoid arthritis: properties and advantages. Ann Rheum Dis. 2001; 60(9): 817-27. PMID: 11502606, PMCID: PMC1753828.

13) Erdes SH. Some aspects of pathogenesis of ankylosing spondylitis. TerArkh. 2011; 83(5): 51-6. PMID: 21780658.

14) Schett G, Rudwaleit M. Can we stop progression of ankylosing spondylitis? Best Pract Res Clin Rheumatol. 2010; 24(3): 363-71. doi: 10.1016/j.berh.2010.01.005. PMID: 20534370.

15) Lories R. The balance of tissue repair and remodeling in chronic arthritis. Nat Rev Rheumatol. 2011; 18; 7(12): 700-7. doi: 10.1038/nrrheum.2011.156. PMID: 22009331

16) Park M, Lee S, Park Y. Correlations of serum bone morphogenetic proteins and transforming growth factor-beta-1 levels with spinal dysmobility and radiographic damage in ankylosing spondylitis. Ann Rheum Dis. 2005; 3(suppl): 312.

17) Kong W, Wang $\mathrm{x}$, Zhou T, Jin Y, TAO Q, Xu Y, et al. Serum levels of bone morphogenetic protein-7 and Sclerostin are elevated in ankylosing spondylitis, but not linked with structural damage. Arthritis and Rheumatology. 2014; 66(10): 1129.

18) Park M, Chung S, Park Y, Lee S. Suppression of bone morphogenetic proteins attenuates syndesmophytosis by down-regulating smad pathway in aggrecan-induced spondylitis mice. Arthritis Rheum. 2008; 58: 346.

19) Wendling D, Cedoz JP, Racadot E. Serum levels of MMP-3 and cathepsin K in patients with ankylosing spondylitis: effect of TNF alpha antagonist therapy. Joint Bone Spine. 2008; 75(5): 559-62. doi: 10.1016/j.jbspin.2008.01.026. PMID: 18674944.

20) Son JH, Cha SW. Anti-TNF-alpha therapy for ankylosing spondylitis. Clin Orthop Surg. 2010; 2(1): $28-33$. doi: 10.4055/cios.2010.2.1.28. PMID: 20190998, PMCID: PMC2824092.

21) Jacobs WB, Fehlings MG. Ankylosing spondylitis and spinal cord injury: origin, incidence, management, and avoidance. Neurosurg Focus. 2008; 24(1): 12. doi: 10.3171/foc/2008/24/1/e12. PMID: 18290738.

22) Klareskog L, Catrina AI, Paget S. Rheumatoid arthritis. Lancet. 2009; 373(9664): 659-72. doi: 10.1016/s0140-6736(09)60008-8.

23) Wendling D, Cedoz JP, Racadot E, Dumoulin G. Serum IL-17, BMP-7, and bone turnover markers in patients with ankylosing spondylitis. Joint Bone Spine. 2007; 74(3): 304-5. doi: 10.1016/j.jbspin.2006.11.005. PMID: 17369068.

24) Vosse D, Landewé R, Garnero P, van der Heijde D, van der Linden S, Geusens P. Association of markers of bone-and cartilage-degradation with radiological changes at baseline and after 2 years follow-up in 
patients with ankylosing spondylitis. Rheumatology (Oxford). 2008; 47(8): 1219-22. doi: 10.1093/rheumatology/ken148. PMID: 18539620.

25) Visvanathan S, van der Heijde D, Deodhar A, Wagner C, Baker DG, Han J, et al. Effects of infliximab on markers of inflammation and bone turnover and associations with bone mineral density in patients with ankylosing spondylitis. Ann Rheum Dis. 2009; 68: 175-182. doi: 10.1136/ard.2007.084426. PMID: 18495735, PMCID: PMC2605572.

26) van der Heijde D, Machado P, Braun J, Hermann KG, Baraliakos X, Hsu B, et al. MRI-inflammation of the vertebral unit (VU) only marginally contributes to new syndesmopyhte formation in that unit: a multi-levelanalysis. Ann Rheum Dis. 2012; 71: 369-73. doi: 10.1136/annrheumdis-2011-200208. PMID: 21979001.

27) Maksymowych WP, Chiowchanwisawakit P, Clare T, Pedersen SJ, Østergaard M, Lambert RG. Inflammatory lesions of the spine on magnetic resonance imaging predict the development of new syndesmophytes in ankylosing spondylitis: evidence of a relationship between inflammation and new bone formation. Arthritis Rheum. 2009; 60(1): 93-102. doi: 10.1002/art.24132. PMID: 19116919.

28) Baraliakos X, Listing J, Brandt J, Haibel H, Rudwaleit M, Sieper J, et al. Radiographic progression in patients with ankylosing spondylitis after 4 yrs of treatment with the anti-TNF-alpha antibody infliximab. Rheumatology (Oxford). 2007; 46(9): 1450-3. doi: 10.1093/rheumatology/kem166. PMID: 17623745.

29) Baraliakos X, Listing J, Rudwaleit M, Sieper J, Braun J. The relationship between inflammation and new bone formation in patients with ankylosing spondylitis. Arthritis Res Ther. 2008; 10(5): 104. doi: 10.1186/ar2496. PMID: 18761747, PMCID: PMC2592781. 